\title{
Reconhecimento precoce e tratamento das fraturas e luxações carpometacarpais
}

\section{Early Recognition and Treatment of Carpometacarpal Fractures and Dislocations}

\author{
Eduardo Murilo Novak ${ }^{1,2}$ Giana Silveira Giostri ${ }^{1}$ \\ ${ }^{1}$ Escola de Medicina, Hospital Universitário Cajuru, Pontifícia \\ Universidade Católica do Paraná, Curitiba, PR, Brazil \\ 2 Departamento de Medicina Forense e Psiquiatria, Universidade \\ Federal do Paraná, Curitiba, PR, Brasil
}

\begin{abstract}
Endereço para correspondência Eduardo Murilo Novak, PhD, Escola de Medicina, Pontifícia Universidade Católica do Paraná, Rua Imaculada Conceição, 1155, B 6, Curitiba, PR, Brasil (e-mail: eduardonovak@gmail.com).
\end{abstract}

Rev Bras Ortop 2021;56(5):537-542.

\author{
Resumo \\ Palavras-chave \\ - articulações \\ carpometacarpais/ \\ lesões \\ - articulações \\ carpometacarpais/ \\ patologia \\ - articulações \\ carpometacarpais/ \\ cirurgia \\ - luxações articulares \\ - ossos da mão
}

As lesões na região carpometacarpal (CMC) podem passar despercebidas em mais da metade dos casos. O diagnóstico precoce é determinante para o tratamento. Embora clinicamente o paciente já possa demonstrar que de fato ocorreu a lesão, radiografias apropriadas, principalmente na incidência em perfil, são fundamentais para o diagnóstico. A lesão mais comum afeta as articulações de $4^{\circ}$ e $5^{\circ}$ dedos. 0 tratamento vai depender do tipo da lesão e o grau de envolvimento das articulações. Geralmente, é necessária redução e fixação.Quando apenas um raio é acometido, habitualmente o $5^{\circ}$, pode ser feita redução fechada e fixação com fios de Kirschner. Nos casos complexos, faz-se necessária a redução aberta e fixação, sendo os fios de Kirschner os materiais mais comumente usados. Após a retirada dos fios, intensifica-se a reabilitação.Se foi alcançada uma redução apropriada, esperam-se resultados funcionais e radiográficos satisfatórios.

Carpometacarpal (CMC) injuries can easily be missed in more than half of the cases. Early diagnosis is crucial for treatment. Although the clinical aspect can lead the treating physician to suspect that anything is going wrong, appropriate radiographs, especially in the lateral view, are crucial for the diagnosis. The most common CMC fracture dislocations affects the $4^{\text {th }}$ and $5^{\text {th }}$ joints. Treatment will depend on the type of injury and on the degree of joint involvement. Reduction and fixation are usually required. When only one ray is affected, usually the $5^{\text {th }}$, closed reduction and fixation with Kirschner wires can be performed. In complex cases, open reduction and fixation are required, with Kirschner wires being the most commonly used materials. After the hardware removal, rehabilitation can be intensified. If an appropriate reduction has been achieved, satisfactory functional and radiological outcomes are expected. recebido

12 de Agosto de 2019

aceito

20 de Fevereiro de 2020

Publicado on-line

Março 30, 2021
DOI https://doi.org/

$10.1055 / \mathrm{s}-0040-1712138$. ISSN 0102-3616. (c) 2021. Sociedade Brasileira de Ortopedia e Traumatologia. All rights reserved.

This is an open access article published by Thieme under the terms of the Creative Commons Attribution-NonDerivative-NonCommercial-License, permitting copying and reproduction so long as the original work is given appropriate credit. Contents may not be used for commercial purposes, or adapted, remixed, transformed or built upon. (https://creativecommons.org/ licenses/by-nc-nd/4.0/)

Thieme Revinter Publicações Ltda., Rua do Matoso 170, Rio de Janeiro, RJ, CEP 20270-135, Brazil 


\section{Introdução}

Embora corresponda a $<1 \%$ das lesões da mão, ${ }^{1}$ não é raro restar a dúvida se há alguma alteração radiográfica na base dos metacarpais (MTCs) do $2^{\circ}$ ao $5^{\circ}$ dedos na rotina do atendimento ao trauma ortopédico. Isso porque há uma sobreposição óssea entre os MTCs e os ossos do carpo, que podem gerar algum equívoco na interpretação das imagens. ${ }^{2}$ Essa dificuldade pode ser mitigada se a avaliação clínica for bem feita e se o conhecimento anatômico for aprimorado.

As articulações na chamada região carpometacarpal (CMC) são em regra rígidas, firmes, estáveis, principalmente nos dedos mais radiais. Traumas leves dificilmente levarão a lesões maiores. Por isso, estabelecer o real mecanismo do acidente e realizar um bom exame físico podem ser determinantes para não negligenciar esse tipo de lesão. Henderson et al. ${ }^{2}$ relataram falha diagnóstica em 15 de 21 pacientes, relacionando a dois principais fatores: o edema, que dificulta um exame mais acurado, e a falta de uma radiografia em perfil.

Assim, se pode haver alguma complexidade no diagnóstico, é de se esperar que o tratamento também tenha seus percalços. E isso de fato ocorre, pois aqueles ligamentos lesados, para retornarem à estabilidade que tinham antes do acidente, precisam ser cicatrizados na posição correta, qual seja, com as articulações relacionando-se perfeitamente entre si, de modo a proporcionar uma mão funcional e sem dor. ${ }^{3}$

Em um primeiro momento, poderia se deduzir que, já que não há mobilidade na base do $2^{\circ}$ e $3^{\circ} \mathrm{MTC}$, então qualquer redução seria aceitável. Isso não é uma verdade, pela simples razão de que o centro da mão recai exatamente sobre $03^{\circ}$ raio, e se este estiver mal reduzido, poderá trazer consequências como diminuição na força de preensão. ${ }^{4}$

O propósito da presente revisão abrangerá aspectos anatômicos e funcionais pertinentes, qual o estado da arte no tocante ao diagnóstico das fraturas e luxações CMCs (Quadro 1) e o que se tem de atualidades referentes aos diversos tipos de tratamento.

\section{Anatomia e Cinesiologia}

Há uma diferença considerável em termos anatômicos entre as bases dos diferentes MTCs, e em razão disso, a amplitude de movimentos em flexo-extensão, apesar de variável, é menor nos $2^{\circ}$ e $3^{\circ}$ do que nos $4^{\circ}$ e $5^{\circ}$ MTCs. Gunther ${ }^{5}$ mostrou, em estudo cadavérico, que não há mais do que $1^{\circ}$ de movimento em flexo-extensão na base do segundo MTC, $3^{\circ}$ no terceiro, $8^{\circ}$ no quarto e $15^{\circ}$ no quinto. Harwin et al. ${ }^{6}$ encontraram $25^{\circ}$ a $30^{\circ}$ para o quinto, $15^{\circ}$ para o quarto, e movimento limitado no segundo e no terceiro.

Há diversos ligamentos que mantêm a estabilidade nessa região. Nakamura et al. ${ }^{7}$ discorreram que no $2^{\circ}$ MTC existem dois ligamentos dorsais e um volar, no $3^{\circ}$ MTC são quatro ligamentos volares e três dorsais, no $4^{\circ}$ MTC existe um ligamento volar e dois dorsais e no $5^{\circ}$ MTC há um volar e dois dorsais, com diferentes variações morfológicas entre eles. Esse maior número de estruturas ligamentares volares e dorsais na base do $3^{\circ}$ MTC contribui para a estabilidade das articulações CMCs adjacentes.

Com relação às articulações, os mesmos autores relataram que entre o $4^{\circ}$ e $5^{\circ}$ MTC, o $2^{\circ}$ MTC e o trapézio, e entre o trapézio e o trapezoide eram todas articulações simples. Havia uma maior variação nas articulações entre o $3^{\circ}$ e $4^{\circ}$ MTC ( 5 tipos: faceta dupla de tamanho igual, faceta pequena dupla dorsal, faceta pequena dupla volar, faceta única apenas dorsal e faceta grande única), entre o $2^{\circ}$ e $3^{\circ}$ MTC (2 tipos: faceta única e faceta dupla), entre o capitato e o hamato ( 3 tipos: faceta única em formato de L, faceta dupla, e faceta simples em formato de 0 ) e entre o trapezoide e o capitato (3 tipos: faceta única volar, faceta dupla e faceta única grande).

\section{Quadro 1 Lesões Carpometacarpais}


Então, sinteticamente o que se tem é as bases do dedo indicador e médio rígidas, em razão da articulação com o trapézio, trapezoide e $3^{\circ}$ MTC, e também pela presença daqueles ligamentos dorsais e volares, em maior número na base do $3^{\circ} \mathrm{MTC}$. Além disso, há inserções dos tendões do flexor radial e dos extensores radiais longo e curto do carpo, nas bases desses MTCs, o que pode inclusive contribuir na avulsão de fragmentos ósseos. 0 estabilizador dinâmico da $5^{a}$ articulação CMC é o tendão do extensor ulnar do carpo, que também contribui para o desvio dorsal nas luxações. ${ }^{3}$

\section{Epidemiologia e Classificação}

Dobyns et al. ${ }^{8}$ reportaram que, entre 1.621 fraturas de mão estudadas, apenas 3 corresponderam a fratura-luxação CMC.

Steinmetz et al. ${ }^{1}$ descreveram que entre 81 lesões, 23 $(28,8 \%)$ corresponderam a lesão isolada na região $\mathrm{CMC}$ do $5^{\circ}$, e 37 (45\%) deveram-se a lesão de $4^{\circ}$ e $5^{\circ}$. No mesmo estudo, $87,5 \%$ dos casos corresponderam a homens, com a mão direita sendo acometida em $78,8 \%$ do total. A maioria dos casos deveu-se a soco com a mão fechada (46,3\%), seguida de queda de altura (20\%) e acidente de trânsito (12,5\%). Fisher et al. $^{9}$ publicaram que múltiplas luxações ocorrem mais frequentemente do que as isoladas. Em $50 \%$ dos casos houve envolvimento isolado da articulação entre o $5^{\circ}$ MTC e o hamato, e $25 \%$ afetaram o $2^{\circ}$ MTC.

Como há estruturas ligamentares bastante fortes, pode haver avulsão e fraturas impactadas concomitantemente às lesões CMCs. As luxações dorsais são mais comuns do que as volares, haja vista que os traumas diretos tendem a forçar o MTC em flexão. Steinmetz et al. ${ }^{1}$ encontraram apenas 1 luxação volar entre 165 casos avaliados.

Há vários sistemas de classificação descritos. ${ }^{10-13}$ Como a maior parte das lesões acomete os raios ulnares, as classificações são mais voltadas para essa área e ressaltam a possível associação com fraturas do hamato. Cain et al. ${ }^{10}$ dividem em tipo $1 \mathrm{~A}$ (subluxação ou luxação do $5^{\circ}$ MTC sem fratura do hamato), tipo 1B (fratura dorsal do hamato, tipo 2 (cominuição dorsal do hamato) e tipo 3 (fratura coronal do hamato). Tay et al. ${ }^{12}$ estratificam como tipo 1 aquelas que envolvem apenas um raio, tipo 2 as que acometem dois raios, porém no subtipo $2 \mathrm{~A}$ a fratura do $4^{\circ}$ MTC é extra-articular e na $2 B$ há luxação ou fraturaluxação de $4^{\circ}$ e $5^{\circ}$, e tipo 3 as que envolvem fratura do hamato. Há uma outra classificação, simples, citada por Pundkare et al., que abrange todas as lesões, porém refere-se apenas à direção do desvio, se dorsal (tipo A), volar (tipo B) ou divergente (tipo C). ${ }^{13}$

Outras lesões menos frequentes também são descritas. A luxação do $2^{\circ}$ e $3^{\circ}$ MTC, volar ou dorsal, tem sido relatada na literatura. ${ }^{14}$ Zaizi et al. ${ }^{15}$ publicaram um caso de luxação divergente, em que o $3^{\circ}$ MTC deslocou-se para volar e o $2^{\circ}$ MTC luxou para a região dorsal. Mane et al. ${ }^{16}$ mostraram uma lesão não diagnosticada numa paciente que teve luxação volar das cinco articulações CMCs.

\section{Suspeita Clínica}

A avaliação clínica inicial é de suma importância para a identificação da lesão. Deve-se atentar primeiramente à história relatada referente ao mecanismo de trauma. Geralmente o paciente narra um impacto direto na mão, com quantidade de energia cinética significativa. Na grande maioria das vezes, o relato é de trauma com o punho cerrado como nas agressões com socos -, acidentes automobilísticos e quedas. De rotina, deve ser sempre indagado se houve um acidente prévio, pois não raro, por absoluta falha técnica na anamnese, o médico só descobre que existia uma lesão antiga quando tenta de todo modo obter a redução e percebe que não é possível. ${ }^{2}$

o exame físico revela dor, impotência funcional e aumento de volume. 0 edema pronunciado dificulta a palpação óssea dorsal e a percepção de descontinuidade entre a base dos MTCs e a fileira distal dos ossos do carpo. Vencendose essa etapa, ou seja, suspeitando-se de que algo não está a contento, parte-se para o exame radiográfico. Aqui talvez seja o principal momento em que um erro pode ocorrer, pois identificar a alteração, principalmente nos dedos ulnares, é difícil. Mais da metade das lesões nessa região pode passar despercebida num primeiro atendimento, em que o foco da atenção é frequentemente desviado em virtude de existirem outras lesões mais impactantes ao suporte de vida do paciente. $^{17}$

\section{As Imagens}

A anatomia radiográfica da região $\mathrm{CMC}$ parece, numa primeira vista, simples de se compreender. Do $2^{\circ}$ ao $5^{\circ}$ ocorre a articulação com trapézio, trapezoide, capitato e hamato. Porém, como há sobreposição das linhas na imagem bidimensional, nem sempre é possível detectar-se, de pronto, que houve uma lesão, principalmente naqueles casos em que há acometimento isolado em algum dos raios, como o $5^{\circ}$.

Fisher et al. ${ }^{9}$ descreveram o paralelismo que deve haver nas superfícies articulares da segunda fileira do carpo (- Figura 1). Na radiografia de frente, caso já seja identificada uma ausência dessa congruência, já deve ser suspeitada a lesão.

Além disso, na incidência de frente, deve-se prestar atenção às linhas da cascata MTCs, conforme descritas por Hodgson et al. ${ }^{18}$ Esses autores relataram que as linhas do eixo central de cada MTC convergem a um ponto comum, localizado a cerca de

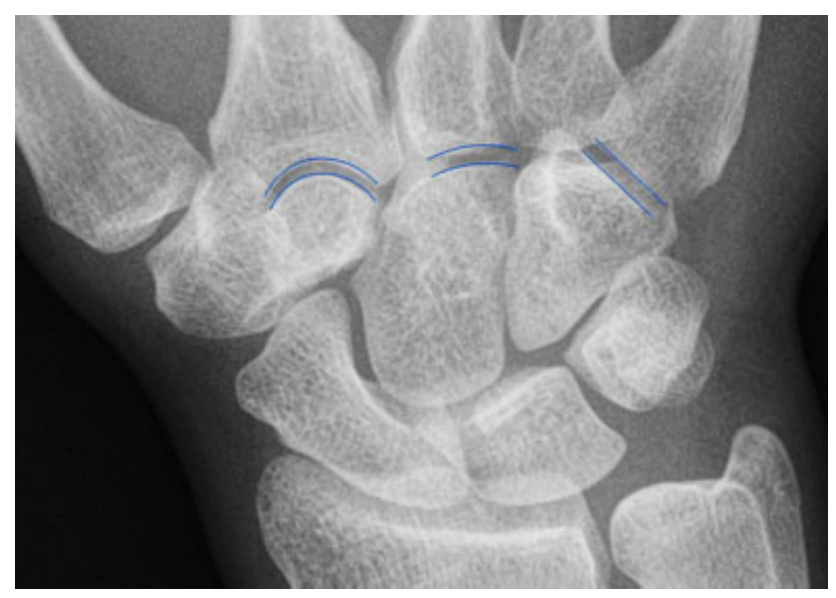

Fig. 1 Radiografia de frente da região do punho normal, mostrando o paralelismo entre as superfícies articulares. 
dois centímetros proximal à superfície articular do rádio. Se algum dos MTCs estiver subluxado ou luxado, então não haverá essa convergência de todos os raios.

Um dos pontos que talvez possa ser causa de interpretação errônea das lesões é a solicitação rotineira de radiografia da mão nas projeções frente e oblíqua. Como o paciente relata um trauma na mão, então o impulso inicial é o médico não se atentar a solicitar o perfil, e satisfazer-se apenas com essas duas incidências. Dessa forma, se não estiver familiarizado com a anatomia radiográfica do punho visto de frente, poderá deixar passar uma lesão na base dos MTCs. Daí a necessidade ao menos da incidência lateral. ${ }^{19}$ Nessa incidência, ainda, é possível avaliar-se o ângulo intermetacarpal, conforme descrito por McDonald et al. ${ }^{20}$ Esses autores demonstraram que, nas lesões MTCs do lado ulnar do carpo, os ângulos diafisários entre o $2^{\circ}$ e o $5^{\circ}$ MTC e entre o $3^{\circ}$ e $5^{\circ}$ MTC geralmente são $>10^{\circ}$; e ressalvaram que para esses casos há indicação de exames de imagens avançados.

Além dessas projeções, há outras possibilidades. Lefere et al., ${ }^{21}$ ao descreverem uma sistematização para análise radiográfica do punho, preconizaram solicitar PA, perfil e oblíquas. Em PA, lembraram que deve-se observar o alinhamento dos arcos carpais e ausência de sobreposição de superfícies articulares.Nas suspeitas de acometimento CMC, as oblíquas serviriam para analisar com mais detalhes as luxações. O perfil seria destinado a verificar o alinhamento rádio-semilunar-terceiro MTC.

Requisitar tomografia computadorizada (TC) pode ser uma opção para resolver os casos duvidosos, em que a associação entre mecanismo de trauma, exame físico criterioso e radiografias nítidas em incidências apropriadas não é suficiente para apontar o diagnóstico ou mesmo esclarecer se há fragmentos ósseos articulares. Steinmetz et al., ${ }^{1}$ em sua análise de 80 pacientes com esse tipo de lesão, relataram que não houve necessidade desse tipo de exame. Por outro lado, Talmaç et al. ${ }^{4}$ fizeram uso do exame para todos os 14 pacientes avaliados.

\section{Tratamento}

O objetivo principal de qualquer terapêutica é restaurar a função da mão, sem dor, prevenindo degeneração articular pós-traumática. $\mathrm{O}$ tratamento das lesões CMCs vai depender de uma série de elementos, como área e estruturas acometidas, grau de deslocamento, atividade do paciente, não existindo um algoritmo uniforme de tratamento, principalmente em razão da baixa frequência desse tipo de trauma. ${ }^{3} \mathrm{~A}$ modalidade terapêutica aceita varia desde fixação intramedular ou transversa com fios de Kirschner (K) até fixação com placas de apoio dorsais. ${ }^{12,22} \mathrm{Um}$ estudo comparou placa e parafusos com fixação com fios de $K$, concluindo que a fixação com fios é o padrão-ouro para o tratamento das lesões de $4^{\circ}$ e $5^{\circ}$ metacarpais. ${ }^{23}$ Artroscopia também tem sido descrita como método auxiliar. ${ }^{24}$

Nas luxações isoladas, passíveis de redução fechada, a estabilização com fios transversos apoiados no metacarpal não lesado podem ser suficientes para promover a estabilização e o tratamento adequado. ${ }^{25}$ Entretanto, Cobb et al., ${ }^{26}$ numa revisão sistemática, afirmaram que as evidências clínicas são exíguas para poder se concluir qual o melhor método de tratamento para as fraturas-luxações do $5^{\circ} \mathrm{MTC}$. Há alternativas, entretanto, à fixação com fios de K. Nishimura et al. trataram 10 pacientes que se apresentaram com luxações de raios ulnares MTCs associadas a fratura cominutiva da base, por meio de fixador externo, concluindo que todos os pacientes retornaram ao nível de função de antes do acidente. $^{27}$

Nos casos de lesões múltiplas, indica-se a cirurgia aberta e a fixação, haja vista a dificuldade de se obter redução fechada adequada em razão da interposição de partes moles. ${ }^{3}$ Nessas luxações que envolvem do $2^{\circ}$ ao $5^{\circ}$ MTC, a chave para a redução é o $3^{\circ}$ MTC junto ao capitato. ${ }^{4}$ Como está no centro, se não estiver bem posicionado, fará com que todos os demais permaneçam em posição inadequada.

Não há consenso sobre o melhor tratamento para essas lesões múltiplas. Na literatura, há descrições desde redução fechada com imobilização gessada até artrodese. ${ }^{28}$ Porém, a tendência da imobilização gessada pós-redução fechada é resultar em subluxação, principalmente em razão da interposição ligamentar. ${ }^{29,30}$ Por isso, conforme mencionado, recomenda-se a redução aberta para os casos de luxações múltiplas, vez que isso diminui o risco de síndrome compartimental e de redução inadequada. ${ }^{3,4}$

Quando há presença de fratura no carpo, pode ser que a redução se torne mais difícil. Por exemplo, o hamato, quando fraturado, pode dificultar o alinhamento articular, demandando então uma redução aberta mais cuidadosa. Isso é possível por meio de um acesso longitudinal, identificando vasos e principalmente isolando os ramos sensitivos do nervo radial e ulnar dorsal. Afastam-se os tendões, expõese a cápsula e promove-se sua abertura, já deixando planejadas as bordas para posterior fechamento.A seguir, identificam-se as lesões, faz-se a redução e a fixação.

Algumas vezes, o tratamento instituído inicialmente pode não ter sido satisfatório, e uma revisão pode ser necessária. Na - Figura 2, pode-se ver a sequência de uma luxação múltipla volar, a fixação inicial que não se mostrou adequada, e a revisão da fratura, com a correta redução e fixação com múltiplos fios de $\mathrm{K}$.

Uma vez concluído o procedimento cirúrgico, mantém-se uma tala que pode ser removida para limpeza ao redor dos fios de K. Radiografias semanais são feitas para conferir a manutenção da redução. Desde o início, instrui-se o paciente a mover os dedos, conforme a dor permita, e a manter a mão elevada, para redução do edema. Essa reabilitação precoce favorece a recuperação em longo prazo, principalmente das articulações metacarpofalângicas. Após a retirada do material de síntese, ao redor de 6 semanas, intensifica-se a reabilitação. Conforme ocorre o ganho de amplitude de movimento, encoraja-se o paciente a segurar objetos, inicialmente de pouco peso, progredindo conforme a força de preensão gradualmente retorne.

Podem ocorrer complicações, na maioria das vezes decorrentes da gravidade do trauma ou na demora em se iniciar o tratamento. Dependendo do local em que se está, pode ser que o paciente se apresente algumas semanas após o 


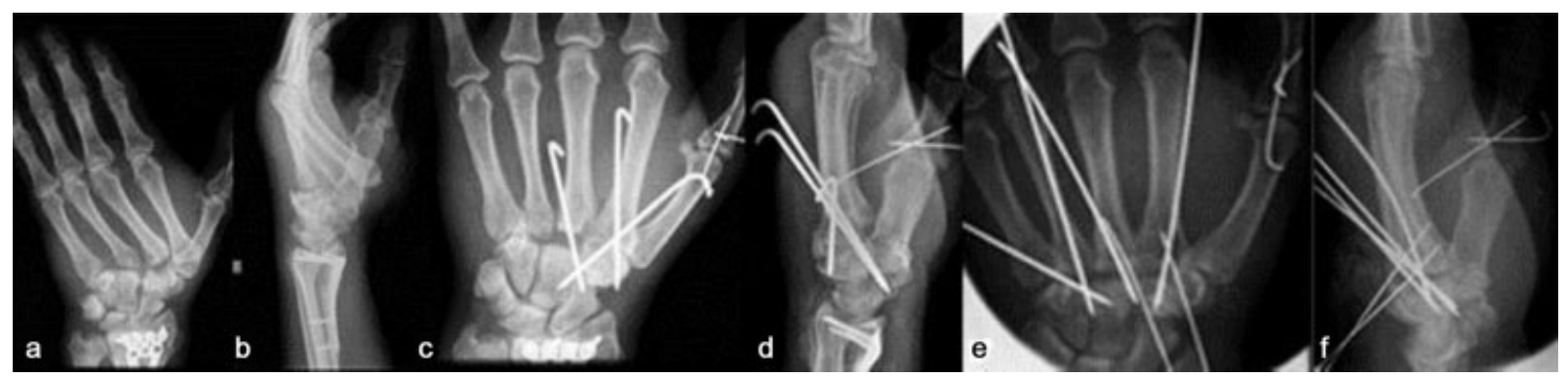

Fig. 2 Luxação volar de $2^{\circ}$ ao $5^{\circ}$ metacarpal. A e B radiografias iniciais, mostrando a lesão volar; C e D evidenciando o primeiro tratamento, em que se percebe a incongruência articular, tanto na radiografia de frente quanto na de perfil; C e D mostram a correta correlação articular.

acidente, e isso dificulta a terapêutica. Em outros casos, a falha diagnóstica pode ser a responsável pelo atraso. Lesões concomitantes, como lesão do nervo ulnar, também podem ser responsáveis por maus resultados. Fixação inadequada pode levar à perda de redução, e necessidade de nova cirurgia. Infecção pode resultar em degeneração articular. Enfim, os casos de insucesso no tratamento podem culminar em artrose pós-traumática, vindo a se fazer necessária uma artrodese no futuro. ${ }^{3}$ Nos casos de quadro crônico sintomático da base do $5^{\circ}$ MTC, uma alternativa é a realização da técnica de Dubert, em que é feita uma ressecção da base do MTC, associada a confecção de uma sinostose junto ao $4^{\circ}$ MTC. ${ }^{31}$ Para evitar tais complicações, uma vez que se tenha o diagnóstico de fraturas ou luxações CMCs, a redução precoce, a estabilização adequada e a reabilitação tão logo seja possível devem ser implementadas.

\section{Sistematização do Atendimento}

Como rotina e sistematização de abordagem às lesões CMCs pode-se estabelecer o que segue. Inicialmente, nas lesões traumáticas, o esqueleto apendicular deve ser sempre examinado, a fim de evitar lesões que possam passar despercebidas, principalmente no paciente com perda da consciência. Uma vez identificadas as alterações ectoscópicas na mão, a palpação poderá revelar a crepitação na região. A confirmação se dá com a radiografia, e deve-se atentar para a incidência em perfil, em que se observa principalmente o alinhamento do $3^{\circ}$ MTC. Tomografia computadorizada pode ser solicitada, em especial nos casos de dúvidas diagnósticas ou da extensão do comprometimento articular. Havendo lesão isolada, é possível a redução incruenta e fixação com fios de K ao MTC íntegro adjacente. Nas lesões múltiplas, a redução aberta permite uma melhor visualização da congruência articular.

Em nossa experiência, os resultados deixam de ser satisfatórios quando a lesão não é reconhecida no primeiro tempo, ou se a redução não é obtida integralmente, permanecendo subluxação ou luxação de um ou mais MTCs. Para evitar que isso ocorra, as imagens do intensificador devem ser vistas em múltiplos planos, dando principal atenção à projeção lateral. Outro elemento que debilita os resultados é a dificuldade para reabilitação, pois o paciente deve ser encorajado a mover os dedos tão logo o edema regrida e a dor permita. Atrasar o início da movimentação pode trazer rigidez dos dedos. Também, os casos em que a imobilização pós-operatória foi inadequada, por dificuldade de obtenção da flexão das articulações metacarpofalângicas, mantendoas estendidas, trarão problemas futuros para a função de preensão da mão.

\section{Considerações Finais}

As lesões CMCs podem passar despercebidas, principalmente se não for dada a devida atenção ao mecanismo de trauma e se a avaliação radiográfica não for acurada. Deve-se conhecer a anatomia articular para que se possa fazer o pronto diagnóstico. A radiografia em perfil pode ser a chave para a detecção dessas lesões. Uma vez diagnosticada, a lesão CMC deve ser tratada tão logo estejam reunidas as condições para tal. Lesões isoladas podem ser manejadas com fixação percutânea, ancorando-se o MTC acometido no adjacente íntegro. Lesões múltiplas geralmente requerem redução aberta e fixação. O material de síntese mais comumente utilizado, com bons resultados, é o fio de $\mathrm{K}$, e a imobilização pósoperatória deve ser feita com as articulações metacarpofalângicas em flexão e as interfalângicas em extensão. Desde o início do período pós-cirúrgico, estimula-se o paciente a mover os dedos. Após a retirada dos fios de $\mathrm{K}$, intensificase a reabilitação. Se foi alcançada uma boa redução, é de se esperar que o paciente recupere a força e a função da mão, sem dor. Caso contrário, poderão aparecer alterações degenerativas, que demandarão no futuro novas intervenções, como artrodese.

Nota

O presente artigo de revisão não faz necessária a submissão ao Comitê de Ética em Pesquisa.

Conflito de Interesses

Os autores declaram não haver conflito de interesses.

\section{Referências}

1 Steinmetz G, Corning E, Hulse T, et al. Carpometacarpal FractureDislocations: A Retrospective Review of Injury Characteristics and Radiographic Outcomes. Hand (N Y) 2019:1558944719852743

2 Henderson JJ, Arafa MA. Carpometacarpal dislocation. An easily missed diagnosis. J Bone Joint Surg Br 1987;69(02):212-214

3 Büren C, Gehrmann S, Kaufmann R, Windolf J, Lögters T. Management algorithm for index through small finger carpometacarpal fracture dislocations. Eur J Trauma Emerg Surg 2016;42(01):37-42 
4 Talmaç MA, Görgel MA, Dırvar F, Tok O, Özdemir HM. Functional and radiological outcomes of multiple dorsal carpometacarpal fracture dislocations treated with open reduction and internal fixation. Eklem Hastalik Cerrahisi 2019;30(02):130-136

5 Gunther SF. The carpometacarpal joints. Orthop Clin North Am 1984;15(02):259-277

6 Harwin SF, Fox JM, Sedlin ED. Volar dislocation of the bases of the second and third metacarpals. A case report. J Bone Joint Surg Am 1975;57(06):849-851

7 Nakamura K, Patterson RM, Viegas SF. The ligament and skeletal anatomy of the second through fifth carpometacarpal joints and adjacent structures. J Hand Surg Am 2001;26(06):1016-1029

8 Dobyns JH, Linscheid RL, Cooney WP 3rd. Fractures and dislocations of the wrist and hand, then and now. J Hand Surg Am 1983;8 (5 Pt 2):687-690

9 Fisher MR, Rogers LF, Hendrix RW. Systematic approach to identifying fourth and fifth carpometacarpal joint dislocations. AJR Am J Roentgenol 1983;140(02):319-324

10 Cain JE Jr, Shepler TR, Wilson MR. Hamatometacarpal fracturedislocation: classification and treatment. J Hand Surg Am 1987;12 (5 Pt 1):762-767

11 Kim JK, Shin SJ. A novel hamatometacarpal fracture-dislocation classification system based on CT scan. Injury 2012;43(07): 1112-1117

12 Tay SC, Leow MQH, Tan ES. Use of dorsal buttress plate fixation for ulnar carpometacarpal joint fracture dislocations for early mobilization: outcomes of 11 cases. Musculoskelet Surg 2019;103(01):77-82

13 Pundkare GT, Deshpande SS. Proposal for a Radiological Classification System for Carpo-Metacarpal Joint Dislocations with or without Fractures. Malays Orthop J 2018;12(02):42-46

14 Silk G, Vetharajan N, Nagata H. Volar dislocation of the second and third carpometacarpal joints - the Lisfranc injury of the hand? Hand Surg Rehabil 2018;37(05):320-323

15 Zaizi A, El Yaacoubi T, El Bahraouy A, et al. Pure divergent dislocation of the index and middle finger carpometacarpal joints: A rare case. Trauma Case Rep 2019;23:100222

16 Mane PP, Bhat AK, Acharya A. Post-traumatic wind swept deformity: five volar carpometacarpal dislocations. BMJ Case Rep 2019;12(05):e229561

17 Potini VC, Gibson PD, Wu K, Li K, Tan V. A Novel Screening Technique for Ulnar-Sided Carpometacarpal Dislocations. Orthopedics $2017 ; 40(02):$ e352-e356
18 Hodgson PD, Shewring DJ. The 'metacarpal cascade lines'; use in the diagnosis of dislocations of the carpometacarpal joints. J Hand Surg Eur Vol 2007;32(03):277-281

19 Gaheer RS, Ferdinand RD. Fracture dislocation of carpometacarpal joints: a missed injury. Orthopedics 2011;34(05):399

20 McDonald LS, Shupe PG, Hammel N, Kroonen LT. The intermetacarpal angle screening test for ulnar-sided carpometacarpal fracture-dislocations. J Hand Surg Am 2012;37(09):1839-1844

21 Lefere M, Dallaudière B, Omoumi P, Cyteval C, Larbi A. Rare carpometacarpal dislocations. Orthop Traumatol Surg Res 2016; 102(06):813-816

22 Iwata N, Komura S, Hirakawa A, et al. Dorsal buttress plate fixation for the treatment of fracture-dislocation of the fifth carpometacarpal joint with avulsion fracture of the hamate: a case report. Arch Orthop Trauma Surg 2019;139(01):135-139

23 Bao B, Zhu H, Zheng X. Plate versus Kirschner wire fixation in treatment of fourth and fifth carpometacarpal fracture-dislocations: A retrospective cohort study. Int J Surg 2018;52:293-296

24 Slutsky DJ. Arthroscopic reduction and percutaneous fixation of fifth carpometacarpal fracture dislocations. Hand Clin 2011;27 (03):361-367

25 Slocum AMY, Lui TH. Isolated first carpometacarpal joint dislocation managed with closed reduction and splinting. BMJ Case Rep 2019;12(03):e228715

26 Cobb WA, Dingle L, Zarb Adami R, Rodrigues J. Management of fracture-dislocations of the little finger carpometacarpal joint: a systematic review. J Hand Surg Eur Vol 2018;43(05):530-538

27 Nishimura R, Wright L, Seitz WH Jr. Augmented External Fixation of Ulnar Carpometacarpal Joint Fracture Dislocations. Tech Hand Up Extrem Surg 2019;23(02):84-87

28 Hanel DP. Primary fusion of fracture dislocations of central carpometacarpal joints. Clin Orthop Relat Res 1996;(327):85-93

29 Hartwig RH, Louis DS. Multiple carpometacarpal dislocations.A review of four cases. J Bone Joint Surg Am 1979;61(6A):906-908

30 Na KT, Lee SU, Joo SY, Nho JY. Concurrent Index-to-Little Finger Dorsal Dislocations of the Carpometacarpal Joints with Carpal Bone Fractures. Indian J Orthop 2019;53(02):374-376

31 Meraghni N, Bacle G, Marteau E, Bouju Y, Laulan J. Results of the Dubert procedure for chronic painful fracture-dislocations of the fifth carpometacarpal joint. A report of 6 cases. Hand Surg Rehabil 2017;36(05):373-377 